\title{
SECURITY ASSESSMENT CASE STUDIES OF PUBLIC BUILDINGS IN INDIA
}

\author{
MANJARI KHANNA KAPOOR \\ School of Planning \& Architecture, New Delhi, India.
}

\begin{abstract}
Security engineering is a valuable tool in the design of our built environments and almost a compulsory inclusion in some parts of the world. At the same time there is a wide range of approaches and techniques with their own efficacies and vulnerabilities. A lot of the so-called security around us is mere theatrics and many of the urban environment design decisions stem from perceptions and heuristics. A careful scientific analysis and systematic assessment of 'secured environments' helps identify the true merit of many such tools. The aim is to reduce the subjectivity in security and objectify it. Some such experiments with audit of security led us to some startling revelations indicating a direct relationship between security and common sense. We have evidence to deal with the myths and reaffirm the direct relationship between good architectural design and sensible security. The effort is to snatch our built environment from the clutches of the security men and design-in a more wholesome version of security. The idea is to accept and include security as a necessary component, as essential as any other service and integrate it into our built environment seamlessly.
\end{abstract}

KEYWORDS: security, assessment, engineering, crime-prevention, counter-terrorism, designed-in, resilience, organised, mechanical, natural, costs.

\section{INTRODUCTION}

Security is both a physical necessity and a psychological need today. We see a lot happening around us in the name of security from mechanical militarization to placebo theatrics. There is a lot that is technically feasible with growing technological inventions and design innovations. There is lot of literature on these and many guidelines formulated across the world to guide the designers of the urban world.

Perception of the security however is a physiological and psychological necessity but subjective to individual thresholds and cultural predispositions. A doctoral survey by the author lays bare the socio-economic and cultural factors that influence the security perceptions. It establishes a certain link between security perceptions and the cultural predispositions, theological standpoint with a dash of socio-economic markers. This is one of the biggest challenges for the security engineers and potential designers of a secure environment.

As designers and engineers we need measurable objectives, quantifiable efficacy to target and eliminate the eyewash. It is also valuable for justification of project expenses and value based engineering. With the objective of ridding security of its subjectivities, the author's doctoral research undertakes case studies of some highly secured environments (in India). This led to some interesting revelations and greater understanding of the security engineering approaches.

They help us go beyond the perceptible top layer into the deeper realms of well-founded security approach. These case studies reveal a number of factors at play which contribute to security or take away from it intentionally or accidentally. Some of these have larger ramifications for the architectural design paradigm and ideology in general. 


\section{METHODOLOGY}

This paper undertakes close scrutiny of two public buildings in India to study, understand and analyse in depth from the security standpoint. Both the samples are very successful in their security provisions and selected on the basis of:

- high population count or heavy footfalls;

- high threat profiles;

- high security inputs in design and operations;

- high security perceptions attached to the need \& implementation

Based on the above rationale, we identified 2 buildings exemplifying completely different building function and type of security designed. The first is a temple and the second an office complex each with their unique complexities. Their specific details are discussed in the next section.

The systematic methodology for assessment started with some cursory knowledge about these installations, backed by a preliminary literature survey. This was then substantiated with a fair amount of fieldwork including repeated visits to the site, observations and interviews. Simultaneous analysis of the drawings and details entailed a lot of back \& forth. All the assimilated queries were then discussed with the security in charge, policy makers, designers and the project team to get a complete representation of the intent and operations.

All the observations were then carefully analysed for the related factorials. The assessment takes into account the various aspects of security including counter-terrorism, overall crime prevention through environmental design (CPTED) and other related factors like resilience.

The assessment deconstructs the overall security into smaller identifiable components such as perimeter protection, access control, surveillance, vehicular control, MEP design, structural inputs, disaster resilience, design strategies and almost every related aspect as researched. These are then further segregated under the heads of Natural means through design, resilience, inputs aimed at crime prevention and lastly those which are merely serving to feed the perception component without actual effect. This analysis gives an overall picture of the soundness of the security efforts, their appropriateness to the building typology, their cost justifications and efficacy in achieving their security goals.

Please note that Natural security refers to the components which secure by the effective use of design be it spaces, materials or surveillance without the intervention with mechanical (equipment based) or organised (personnel or guards) measures.

So each of the identified buildings was first carefully examined in their layouts and literature survey to get an understanding of the history and context. This, supplemented with interviews with the security designers of the premises help identify the exact list of threats to deal with, which is essential to identify the best strategy in design. Usually this is the best stage to undertake a careful field observation of the working of the building. All questions that arise from the exercise are then addressed by the many interviews with the project team including the architects, structural engineers, MEP designers and the policy makers.

All the collected information is then uploaded and broken down into the 4 categories as listed above to throw up a clear proportion of each aspect at play in securing the premises. Also important is the effectiveness and performance of each of these, derived and analysed in the psycho-social contexts too. 


\section{CASE STUDIES}

\subsection{THE SWAMINARAYAN AKSHARDHAM TEMPLE, New Delhi, India.}

The Akshardham temple in New Delhi is not as much a Hindu temple as it is a spiritualcultural campus. More than its religious relevance, the temple is a centre for tourists to gain awareness of the Indian culture and tradition attracting more than ' $70 \%$ of the tourist population to the capital city' (www.akshardham.com [1]).

\subsubsection{Security context}

The 'Bochasanwasi Shri Akshar Purushottam Swaminarayan Sanstha' (BAPS) owns and patronizes many such temples across India and overseas. While this location was still under construction their campus at Gandhinagar, Gujarat in India was attacked in September 2002 suffering casualties of approx. 30 pilgrims and few security men (Patel [2]).

Traditionally, India is a country with a long history of terrorist attacks and anti-terror security regimens. Also the Hindu-Muslim friction is nothing new especially in the backdrop of the communal tensions that have remained antipathetic ever since the partitioning of the country on theistic basis. Even today, theological wars are raging in the valleys of Kashmir which remains a territorial and a contentious issue for India and its breakaway neighbours accused of harbouring terror for political interests.

In that context, a Hindu temple carries an inherent vulnerability in the current times. Add to that the tourist interest and population numbers. All these continue to put this temple as one of the prime targets in the capital city state. This has made the establishment most aggressively conscious of its security design, sometimes even at the cost of many a tourist getting intimidated by the coldness of the security checks in a place that one would seek calm and inner peace.

\subsubsection{Threats Identified:}

AS PER THE Public Relations Officer (PRO) for the premises, Shri Harish Patel, the chief threat perceived by the administration is 'the unpredictability of the human being' (Patel [2]). This includes human volatility and even small feuds and common crime of any sort that consecrates the environment and spiritual ambience.

AS PER THE AUDIT: Terror, blast, petty crimes, armed attack or hostage situations, bio-chemical attacks, civil unrest, and sabotage in addition to the usual risk of fire and other natural disasters. Being a public place with a huge site setting, gardens etc., women's, kids or seniors' security can be an additional concern depending upon the architectural design.

\subsubsection{Occupants:}

The visitors are the main thrust of this architectural accomplishment including the faithful pilgrim, the curious Hindu outside the immediate cult, the non-Hindu tourist and the International seeker of Indian culture and tradition. The numbers on a given day can go up to a lakh or beyond on national holidays. The staff of the Trust, volunteers, administration and service staff also constitute a sizable population including facilities for their smooth operations. 


\subsubsection{Design overview:}

'The temple, at the centre of the complex, was built according to the Vastushastra and Pancharatra Shasta.The complex features an Abhisheka Mandap, Sahaj Anand water show, a thematic garden and three exhibitions namely Sahajanand Darshan (Hall of Values), Neelkanth Darshan (an IMAX film on the early life of Swaminarayan as the teenage yogi, Neelkanth), and Sanskruti Darshan (cultural boat ride). According to Swaminarayan Hinduism, the word Akshardham means the abode of God and believed by followers as a temporal home of God on earth.' (www.akshardham.com [1])

Designed by an in-house team of volunteers (swamis), a local architect was appointed for the liaison works only. The security also is designed mostly in-house under the guidance of the Head of Security, a retired Brigadier from the NSG.

This temple has been recognised by the Guinness World records as the World's Largest Comprehensive Hindu Temple. The certificate states, 'BAPS Swaminarayan Akshardham in New Delhi, India, is the world's largest comprehensive Hindu temple. It measures $356 \mathrm{ft}$. (109 m). Long, $316 \mathrm{ft} .(96 \mathrm{~m})$. wide and $141 \mathrm{ft}$. (43 m). high, covering an area of 86,342 sq. ft. $\left(8,021.4 \mathrm{~m}^{2}\right)$. The grand, ancient-style, ornately hand-carved stone temple has been built without structural steel within five years by 11,000 artisans and volunteers.' (www.akshardham.com 2017) The complex also houses a guest-house for visiting members, volunteers and swamis. In all there is accommodation for 850 volunteers in approx. 224 family dwelling units' incl. 108 kids (Patel [2]).

The main core is a predominantly single storey structure built as per the Hindu tradition following age-old concepts of site planning which have served well for security and urban design since generations. However, the ancillary functions follow the traditional look with modern technology.

\subsubsection{Site planning:}

The choice and location of site is its greatest element, being quite distant from the main city centre, flanked on two sides by arterial roads maintaining more than 100 metres distance on at least two sides from any of its neighbours. The neighbours too are of a nature that do not contribute to the vulnerabilities and are in turn least affected by the temple itself.

On the balance two sides the temple is served by a Metro station and a bus terminal though well distanced and yet integrated in the urban design. On the 4th side (towards the river banks) lies a residential township which could have been a vulnerability but it is well anticipated in design though it came up a few years after the completion of the temple.

The functions are strategically zoned in layers. This strategy is reminiscent of the traditions from historical monuments like Indian forts and palaces (see Fig. 1 above).

- PERIPHERAL Public zone - public and less secured periphery used for roads and other non-secure peripherals adding to the stand-offs distances from roads.

- SEMI-SECURE Private zone - accessible by authorisation only for Intermediate functions \& backend support.

- SECURE Public zone - this is the main core including the shrine and the human surge at the heart, accessed only after due sanitization and under strict scrutiny and surveillance.

This arrangement of public operations is completely introverted exposing only a limited part of the property to casual scrutiny. Services buildings and staff accommodations are adequately buffered from all public areas with strictly regulated internal traffic circulation. These 


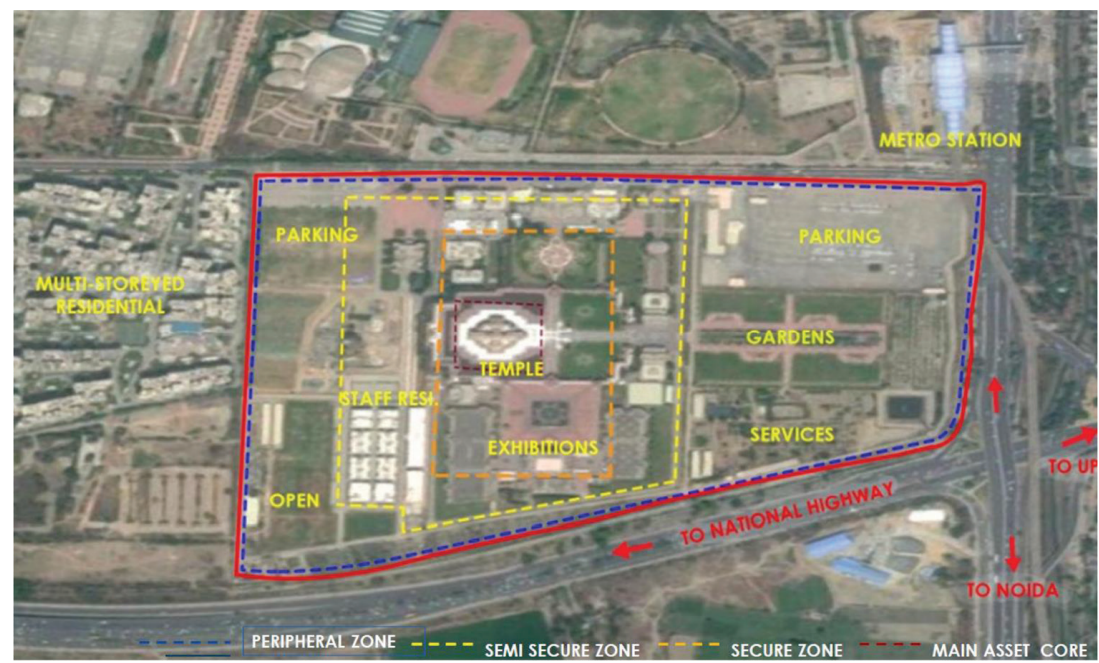

Figure 1: Satellite image of the Akshardham temple (maps [3]).

also add to the protective layer distancing from the side with unknown future development. Parking is strategically distanced from the secured core, on ground but detached.

\subsubsection{Perimeter:}

"Security is not importantly by boxed in walls but mobility of public with assurance of safety. So comfort included in security"- (Patel [2])

Boundary wall (10ft or more) further mounted with a concertina coil (Fig. 2 below) convey the look of an embattlement more than a tourist destination. A more inclusive and transparent perimeter may have been more conducive to the use category but since it is not a part of a larger urban design, it works despite the hostility it generates. The boundary wall supplements the ravine trench on two sides making the severity of the wall seem overdone.

The perimeter is over hardened while it was an opportunity to create a friendlier and magnanimous look and integrate it with the urban design including the Metro station and bus terminus. The hardening does create a sense of suspense and mystique but also an intimidating dent in the peaceful ambience desired and that lies beyond this point.
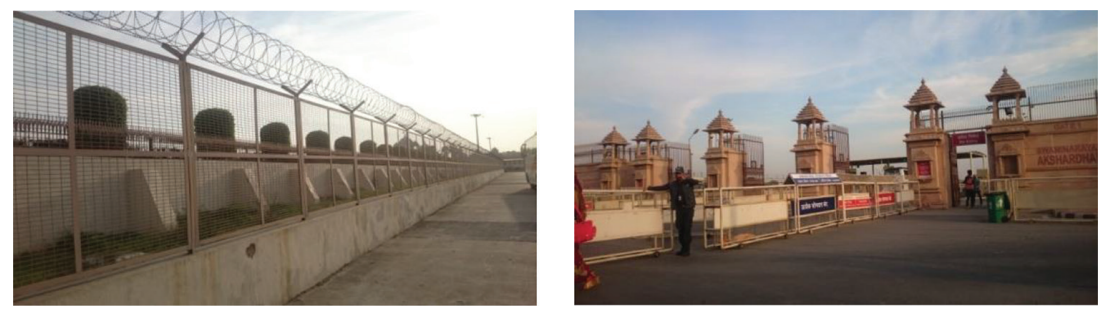

Figure 2 and 3: Boundary wall and gates to the temple complex. 


\subsubsection{Access control:}

Large and sturdy gates are the main measures to control access. They are rendered in the historic and traditional feel (see Fig. 3 above). There are six gates to the complex:

- Gate 1 - open to public vehicles

- Gate 2 - VIP gate only operated for VIP movement

- Gate 3 - service entry for the cafeteria \& guesthouse kitchens

- Gate 4 - entry to the residential part- not accessible to public vehicles

- Gates 5 and 6 - entries from residential block to the temple complex.

These gates are each guarded by personnel $24 \times 7$ and further hardened by boom barriers or tyre busters to protect against forced entry. It may be noted that a large part of these measures are mandatorily stipulated for public places in India. However, in this temple we find them well integrated in design without inconveniencing the visitors or the neighbours.

\subsubsection{Equipment:}

A number of temples and other tourist places in India do not permit carrying phones and other personal belonging due to constraints on checking and security protocols. These restrictions cause all kinds of inconveniences to people creating a parallel terror profile of its own where every individual needs to be forewarned and ready to surrender many of their belongings.

The temple being studied is one of the few that complements its stringent restrictions with amply provided cloakrooms and lockers, free of cost to all and sundry irrespective. 'Security by providing facility- 'decently humanly feasible' (Patel [2]) is part of the BAPS ideology.

These cloakrooms and structures within which the security scanning and frisking takes place, prior to the main entry and temple complex are detached from the rest of the grandeur and built of temporary construction. This makes them seem like an afterthought but actually they fare better due to their low damage potential.

After providing adequate facilities to the visitors the temple administration is very thorough with its scanning, screening and if required frisking to almost the levels followed at international airports. This is a done thing for the Indian tourist and almost a pre-requisite to most locals to feel secure in an environment. However, the research witnessed many tourists getting spurned by the severity at this point. They consider it as a glimpse of further militarization inside and many times opt out, little realising that once the visitor is sanitised the environment inside is one of spirituality and peace.
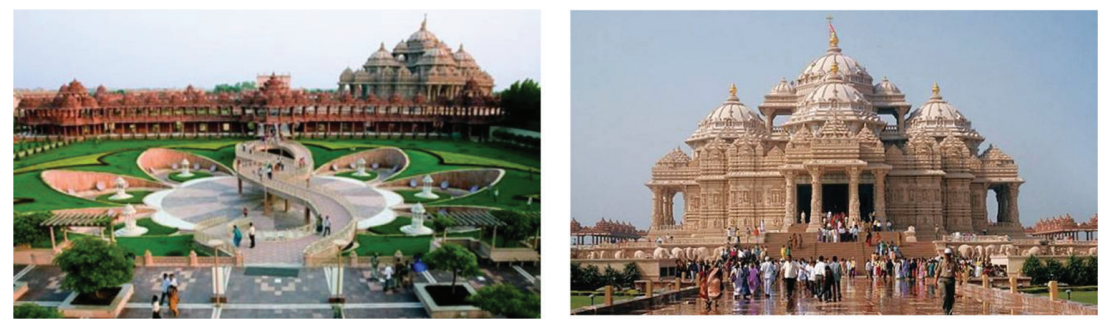

Figures 4 and 5: Traditional style and façade of the temple complex (www.akshardham.com [1]). 


\subsubsection{Surveillance:}

The peripheral areas are open and offer unhindered surveillance by natural and mechanical means. The semi-secure however being triple storeyed needs more mechanical means but the secure zone is laid out in the traditional style offering grand opulent plazas and landscapes which facilitate natural surveillance $24 \times 7$ (see Fig. 4 below). This is further substantiated with a generous distribution of volunteers across the entire complex.

This appears to be an out-dated strategy but the manner and subtlety of its application here actually makes it very appropriate. The volunteers are mostly theistic devotees of the cult, educated and well trained individuals who are not armed security men. So these people while offering casual assistance to the tourist also are the eyes on the street and the secondary function at work discreetly keeping an eye on every activity, foretelling an ugly situation, exercising crowd management, cleanliness and maintenance, all at once.

\subsubsection{Resilience:}

The design is mostly traditional for the Hindu ambience and this too performs well from security view point (see Fig. 5 above). It allows low rise traditional development without structural risks constructed with the historical technology, which has been holding up many old temples for the last many centuries now, even in the face of seismic and other natural forces.

These stone structures have less vulnerability and their external facades are less susceptible to splintering or flying debris. Further the Hindu traditional system follows less openings with the few also screened with jaalis in response to the climate needs. This vocabulary fares extremely well security wise too, offering little insight into the interior functions for better privacy.

The complex is designed in a manner that requires least dependence on the MEP, structure and other disaster strategies of evacuation and egress. This owes largely to the traditional type of layout and construction which further integrate the disaster resilience, fire and life safety codes rather seamlessly. Most other contemporary good practices are well integrated into the operation module which make this tourist destination notch up further on its security audit.

\subsubsection{Main findings of the case study:}

The Akshardham temple is a non-critical public building falling in the medium threat category as per the building type but is treated in design as a medium to high risk category due to high risk perceptions.

The security thought is of a high degree which seems too harsh at places in the context of an otherwise peaceful and calm environment. The complex typifies the psychological play through security design and its appropriate use especially in public places serving cross cultural targets. It needs to accept and understand that globally there can be many interpretations to the same security. At the same time it also exemplifies the universality of the natural means of security as the most effective, non-controversial and non-subjective form.

The traditional approach is a very successful technique to achieve the natural security in planning, finishes, structural and resilient aspects. Even new age concepts of CPTED are well steeped in the traditional process. Essentially the traditional system hinges on simple common sense response to a problem and is hence timeless as it is cost effective. Simple strategies of functional layering, distancing vehicles, seamless screening of huge numbers, their guided management and other covert strategies are found to be much more efficient than the mechanization prevalent in our urban world today. 
3.2 DATA CENTRE COMPLEX for the government of India at Bengaluru, India.

The Government of India undertook the establishment of its Data centres to house the data of the projected 150 million population. Since they would house sensitive data and being a project of national prestige the complexes were perceived as being 'critical', highly sensitive and with a high threat profile. As a result they came to symbolise a Nation's IT prowess and Engineering muscle in the face of the antinationalistic malicious agendas. (Aggarwal [4])

The campus is designed to house a staff of approximately 1000-1500 within, of these about half would be working $24 \times 7$. Being a public utility, it is likely to attract a visitor population of thousands daily. The small museum within is likely to attract busloads of students and tourist excursions. (Grover [5])

\subsubsection{Site planning}

The site selection was not easy in the heart of the burgeoning IT city. Though it is an abundant site, its proximity to a residential colony and access through it creates insurmountable vulnerabilities. Plotted houses touch the site boundary on 1 complete face while 2 faces are vacant for future development. There future use will define another level of threats in the future that cannot be identified now.

The campus is divided into 3 building blocks in order of security concerns (refer Fig. 6 below)-

- Data centre block housing the data storage servers considered the most critical assets.

- Non- data centre block houses the support staff, administrative and public utilities. These are non-critical functions but still high security areas due to the human collateral.

- Utilities block accommodates all services required to make the installation function nonstop. This also has an element of criticality as a breakdown of this support will hinder the critical functioning of the Asset. (Grover [5])

Setbacks and peripheral fire tender driveway is the only vehicular movement The Data centre is tucked back with restricted vehicular movement around it. The strategy to recess the DC block to the rear to layer it and create more distance is exposing it to the vulnerability of the adjoining residential plotted housing. Hence it is designed to neutralise the locational vulnerabilities. (Grover [5]). This block has no basement and is structurally detached. It is

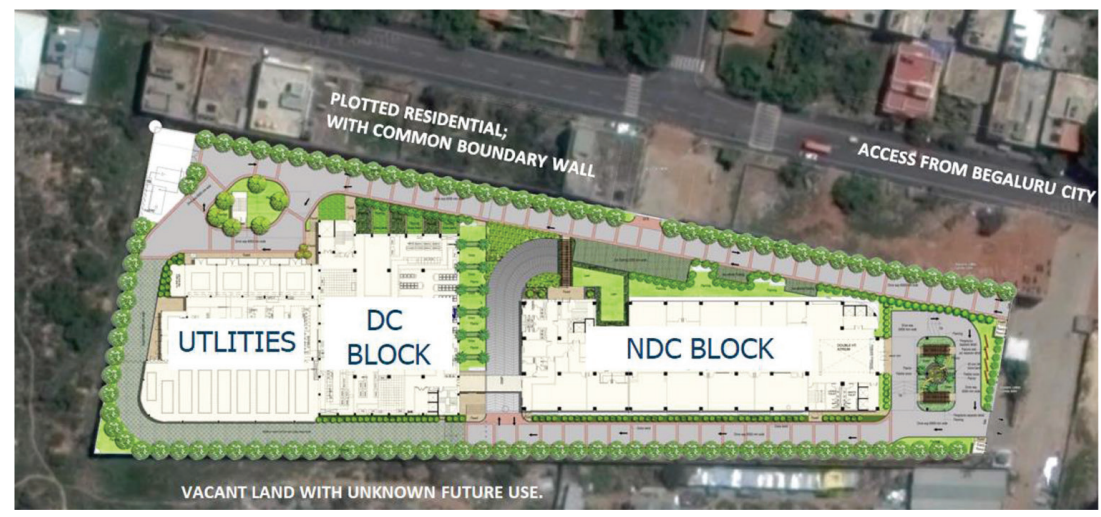

Figure 6: Data centre complex at Bengaluru - layout plan 
rendered as a closed cuboid with the outer skin wall designed as a $600 \mathrm{~mm}$ thick RCC wall, independent of the internal structure with an additional column and slab frame

The NDC block sits on a single basement parking and some services to keep it functional and independent of the critical services block. It houses 6 floors of Tech centre, Regional office, Guest house and accommodation for security men.

\subsubsection{Perimeter, access control and equipment:}

The boundary wall on 2 sides is $3 \mathrm{~m}$ high with a concertina coil. On the front it gets relief with solid steel gates and some relief design and finishing. The 4th side adjoining the neighbours however has a $12 \mathrm{~m}$ high fence atop the boundary wall (see Fig. 8 below). All perimeter openings are further hardened with boom barriers and guard rooms with latest scanners and authorisation facilities.

The peripheral plantation type, height, diameter of foliage is all controlled as per the security inputs. Further some planters and mounds are so created in line with Security strategy to resist forced entries. (Aggarwal [4])

The site has 3 main gates. 2 on the main access face including 1 each for entry and exit. The third one located on the residential face is dedicated to service movement and authorised staff entry only. This gate is provisioned with parking and screening of service vehicles and their cargo. The authorised staffs however are allowed to zip through to reach the basement or the porch where they are intensively screened. (Aggarwal [4])

The visitors use the front gates which are designed to facilitate queuing and space for checking of each and every vehicle, its contents and passengers. The vehicle and its contents are sanitised before admissibility into premises. (Grover [5]) The strategy is meant to neutralise the vulnerability of getting the vehicle into the structure be it the porch or the basement. The spatial constraint necessitates the mechanization of security and maybe justifiable in a critical installation of this nature.

Though most of the invasive checking is done at the gate itself, baggage and personnel screening is mandated at the porch drop-off and the lift entrances in the basements. The vertical circulation within has too much multiplicity of entry and exits thereby exaggerating the security inventory. A lot of the circulation has been curtailed from security considerations.

\subsubsection{Surveillance}

The surveillance strategy is based mostly on cameras of the latest technology and monitoring in the $24 \times 7$ security station located very focally at the lobby level. This unit is operational round the clock and supported by staff dormitories, change rooms, lockers and toilets on the top floor of the NDC block.

Though the NDC block has a glass façade (see Fig. 7 below) and the office spaces inside are designed to look out there is not much spatial scope for surveillance because of the architectural form in the site shape. The DC block is as it is opaque (see rear part in Fig. 7) but the residential neighbours offer an insurmountable vulnerability by offering well concealed vantage points from the outside. The only good part is that here is not much that they can damage directly since the DC is so solid.

\subsubsection{Resilience:}

Resilience is probably the greatest strength of the DC block with high levels of thought to structural, MEP, materials, fire and life safety. The NDC block on the other hand is restricted 

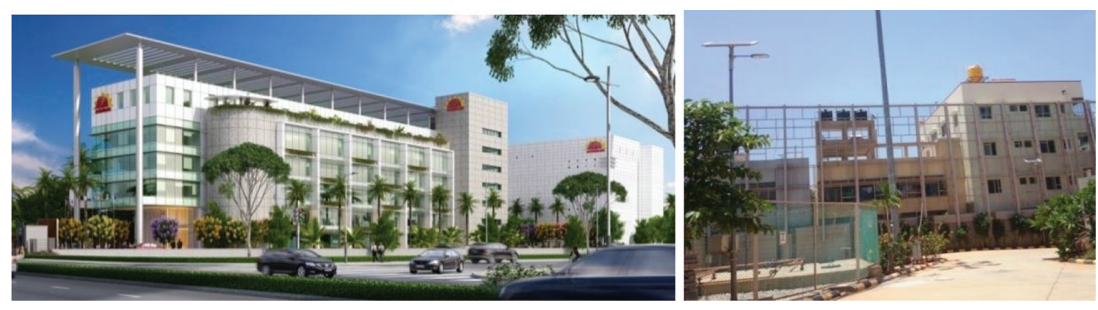

Figure $7 \&$ 8: Data centre complex at Bengaluru - façade and fencing towards residential side.

to only codal compliance to structure, disaster and life safety. The glass is not ideal but still safe enough due to its compliance to green standards. The lamination makes it less dangerous. The granite stone on the façade and other façade finishing materials are regionally sourced but have a high potential to harm.

The DC block however is designed to ensure that even in the worst case scenario the critical operation can continue unabated. The MEP design includes redundancy of all services critical to the data centre including power back-up, air conditioning, lighting et al. The structural hardening includes a 600 thick RCC wall designed to sustain blasts up to $5 \mathrm{~kg}$ of TNT at a distance of $20 \mathrm{~m}$ and detached from the framing of the floor slabs within. The structural design and construction has ensured all joints and structural members are built for the blast shear including even the roof (Singh, 2016) The façade does not have more than 1sqm of openings in keeping with the blast resistant requirements. All doors are shielded by RCC aprons or vestibules. (Singh [6])

\subsubsection{Main findings of the case study:}

The Data centre Complex at Bengaluru is a high risk building requiring high security inputs from the design stage. Despite some unprecedented designed security the assessment shows not very high level overall due to concentration of security thought to the DC block only and some disproportionate dilutions in the NDC block. The DC block in itself is like a bunker while the front shielding block is not indulged even half as much.

The relevance and importance of site selection as a necessary approach is again underscored here. It can escalate or de-escalate a number of vulnerabilities which may or may not be surmountable in operations.

The research uncovers that this building is far from traditional in its rendition and approach. Yet the simple logic of keeping the critical function shielded works well. At the same time while this exposes the DC block to the non-securable, its structural strengthening makes it a worthwhile shield doing well to screen the human lives. So once again we find that good design approach and effective functional zoning can influence security in a much more comprehensive manner than any amount of mechanical and organised means.

This case study exemplifies how the best of budgets and expertise cannot substitute good planning sense and design. Despite the distinction of being the first blast resistant building in India and very high perceptible security, high resilience and high preventive mechanisms, the actual efficacy is not as high due to some inadvertent lapses and insurmountable vulnerabilities. While some vulnerability has been very skilfully addressed by good common sense design strategy, other design lapses remain unaddressed despite a lot of mechanization and militarization. Here too a lot of the security deployment seems to be feeding the associated perceptions for a National icon of this stature. 


\section{CONCLUSIONS}

The case studies show that the traditional approach following the age-old style and planning systems in design invariably serves well from the security perspective. This may be owing to the high priority that security always received in the olden times using the built space as the primary defence tool. Also the natural materials that were used traditionally whether they were sandstone or wood mostly do not have much potential to splinter or create flying debris. They have greater likelihood to absorb the impact. Also most of the traditional approaches sit in extremely well with the CPTED techniques too. The only drawback is the high land requirement and horizontal sprawl it usually entails that can be a constraint in modern times.

The studies also highlight the importance of site selection as one of the most important aspects to consider to maximise advantage from topography and minimise vulnerabilities. Conventional techniques hinge on the basic common sense of layering and securing the core as the most rudimentary wisdom. While the Akshardham temple displayed its optimum use for high embedded security, the Data centre complex demonstrates the constraints and responses of making do with a given site as a practical contemporary reality.

Another important derivative is the huge disconnect between perceptions and actuality of security. The highly secured 'blast resistant structures' for the Data centre complex, are dealing with some big vulnerabilities at heavy initial and running costs. Likewise the very part of the Akshardham temple which seemed to be the highlight of its security (the high level of screening, scanning and restrictions) is effectively its biggest drawback in an otherwise superior design with subtlety. The tourists who find this militarisation at the entry so intimidating that they don't go beyond, miss out on a campus of extreme tranquillity and spirituality far removed from the worldly fears.

Both the projects were found to be very high on the resilience aspects for their own unique design considerations. This is also owing to the local and National codes that put heavy impetus on designing for natural disasters, fire and life safety. These are mandatory stipulations enforced as pre-requisites for all constructional projects. This exemplifies the effectiveness of local stipulations in creating better environments and better guidance to the designers even if it is per force. At the same time the study also shows the unmindful compliance with security mandate even at the cost of creating insecurities.

The singular greatest achievement of the audit is the dramatic difference in the ambience which comes from reduced mechanized and jarring security and substitution by other means. These may range from structural strengthening (as in the Data centre complex) to the helpful and covert deployment of volunteers to supplement the natural means. In the case of the Akshardham temple these were compulsions of the building function but they exemplify that it can be done for any public domain if the dictat so demands.

\section{ACKNOWLEDGEMENTS}

1. Dr. (Prof.) Mandeep Singh, Dean, School of Planning and Architecture and the guide for the Research.

2. Shri Harish Patel, Public Relations officer, Askhardham Temple, New Delhi.

3. Shri P.N. Aggarwal, Retd. DG, Delhi Police and current Security Advisor, Govt. of India.

4. Rajinder Kumar Associates, Architects for the Data Centre complex, New Delhi

5. TPCL Structural engineers for the Data Centre complex, New Delhi. 


\section{REFERENCES}

[1] Swaminarayan Akshardham, BAPS Swaminarayan Sansthan, New Delhi. Online. www.akshardham.com. Accessed on 7 January 2017.

[2] Patel, Shri Harish, Personal communication, 18 February 2017, Public relations officer, Akshardham Temple, New Delhi.

[3] Google map of Akshardham temple, New delhi India. Online. www.googlemaps. Accessed on 20 February 2017.

[4] Aggarwal, P. N., (Retired Director General of Delhi Police). Personal communication, 27 January 2014, Security advisor to Government of India.

[5] Grover, Manika, Personal communication, 10 September 2016, Project Architect, RKA Architects New Delhi, India.

[6] Singh, Er. Amritpal, Personal communication, 8 September 2016, Structural engineer, TPC engineers, Noida, India. 